\title{
CTA Assessment of Coronary Atherosclerotic Plaque Evolution after BVS Implantation - a Follow-up Study
}

\author{
Ioan Ferenț1,2,3, András Mester1,2,3, Monica Chițu1,2,3, Annabella Benedek1,2,3, Mihaela Rațiu1,2, \\ Roxana Hodas $1,2,3$, Imre Benedek $1,2,3$ \\ ${ }^{1}$ University of Medicine and Pharmacy, Tîrgu Mureș, Romania \\ ${ }^{2}$ Center of Advanced Research in Multimodality Cardiac Imaging, Cardio Med Medical Center, Tîrgu Mureș, Romania \\ ${ }^{3}$ Cardiac Critical Care Unit, Clinic of Cardiology, County Clinical Emergency Hospital, Tîrgu Mureș, Romania
}

\section{CORRESPONDENCE}

\section{András Mester}

Str. Gheorghe Marinescu nr. 38

540139 Tîrgu Mureș, Romania

Tel: +40 265215551

E-mail: andras.mester@yahoo.com

\section{ARTICLE HISTORY}

Received: August 15, 2018

Accepted: September 6, 2018
Ioan Ferenț • Str. Gheorghe Marinescu nr. 38, 540139 Tîrgu Mureș, Romania. Tel: +40 265215 551, E-mail: ionutferent2013@gmail.com

Monica Chițu • Str. Gheorghe Marinescu nr. 38 , 540139 Tîrgu Mureș, Romania. Tel: +40 265215 551, E-mail: iulia.chitu@yahoo.com

Annabella Benedek • Str. Gheorghe Marinescu nr. 38 540139 Tîrgu Mures, Romania. Tel: +40 265215 551, E-mail: annabell.benedek@yahoo.com

Mihaela Rațiu • Str. Gheorghe Marinescu nr. 38, 540139 Tîrgu Mureș, Romania. Tel: +40 265215 551, E-mail: d_a_mihaela@yahoo.com

Roxana Hodas • Str. Gheorghe Marinescu nr. 38 540139 Tîrgu Mures, Romania. Tel: +40 265215 551, E-mail: roxana.hodas@yahoo.ro

Imre Benedek • Str. Gheorghe Marinescu nr. 38 540139 Tîrgu Mureș, Romania. Tel: +40 265215551 E-mail: imrebenedek@yahoo.com

\section{ABSTRACT}

Background: Computed tomography angiography (CTA) occupies an important place in the evaluation of coronary atherosclerotic lesions, both before and after the implantation of bioresorbable stents (BVS), providing an accurate assessment of the treated lesions. Aim of the study: This study aims the prospective follow-up of atherosclerotic plaques electively treated with BVS implantation via CTA evaluation in terms of morphological and virtual histology aspects. Material and methods: This is a prospective observational study which enrolled 30 patients electively treated with BVS implantation, in whom CTA was performed after PTCA in order to assess the morphological and virtual histology aspects of coronary plaques. In order to evaluate the impact determined by pre- and post-implantation procedures, statistical analysis was performed among 6 subgroups. Results: After BVS implantation, a significant reduction was observed in terms of stenosis \% (61.63 $\pm 12.63 \%$ in subgroup $1 \mathrm{~A}$ vs. $24.41 \pm 12.48 \%$ in subgroup $1 \mathrm{~B}, \mathrm{p}<0.0001)$ and eccentricity index $(0.46 \pm 0.24$ in subgroup $1 \mathrm{~A}$ vs. $0.43 \pm 0.24$ in subgroup $1 \mathrm{~B}$, $\mathrm{p}<0.0001$ ). In terms of plaque components, there were significant differences with regard to lipid volume and lipid \% (20.07 $\pm 15.67 \mathrm{~mm}^{3}$ in subgroup $1 \mathrm{~A}$ vs. $11.05 \pm 10.83 \mathrm{~mm}^{3}$ in subgroup $1 \mathrm{~B}, \mathrm{p}=$ 0.01), which presented a significant reduction after BVS implantation. The calcium score evaluated locally (82.97 \pm 107.5 in subgroup $1 \mathrm{~A}$ vs. $96.54 \pm 85.73$ in subgroup $1 \mathrm{~B}, \mathrm{p}=0.25)$ and on the target coronary artery (148.2 \pm 222.3 in subgroup $1 \mathrm{~A}$ vs. $206.6 \pm 224.0$ in subgroup 1B, $p=0.10$ ), as well as the total calcium score (377.6 \pm 459.5 in subgroup $1 \mathrm{~A}$ vs. $529.5 \pm 512.9$ in subgroup $1 \mathrm{~B}$, $p=0.32$ ), presented no significant differences when compared with and without post-dilatation lesions. As far as CT vulnerability markers are concerned, the study groups presented significant differences only in terms of spotty calcifications (66.66\% in subgroup $1 \mathrm{~A}$ vs. $79.16 \%$ in subgroup $1 B, p=0.05)$ and low attenuation (37.5\% in subgroup $1 A$ vs. $20.83 \%$ in subgroup $1 B, p=0.01$ ). Conclusions: Following the analysis of coronary artery plaques after the implantation of BVS, significant changes were noted both in the morphology of the atherosclerotic plaques treated with these devices and in the lumen and coronary wall.

Keywords: bioresorbable stent, CTA, elective treatment, coronary artery plaque 


\section{INTRODUCTION}

Coronary computed tomography angiography (CTA) occupies an important place in the evaluation of coronary atherosclerotic lesions, both before and after the implantation of coronary bioresorbable vascular scaffolds (BVS). ${ }^{1}$ This noninvasive examination, still more used as a complementary examination in patients with coronary artery disease next to invasive coronary angiography, offers major benefits in tracking bioresorbable stents.

Having the advantage of radiolucency and resorption over time, a BVS can be accurately evaluated with CTA after implantation, thus opening up a new era in the followup methods of these devices. ${ }^{2,3}$

Due to the possibility of evaluating BVS through coronary CTA, it is feasible to analyze the plaques and their structure through a post-processing software, dedicated to the analysis of atherosclerotic plaque composition, enabling the concurrent analysis of vulnerability characteristics as well as of the information related to the lumen and walls of the coronary arteries. ${ }^{4}$ Details of plaque composition before stenting and its morphological characteristics under the implanted BVS can be obtained after the post-processing of CTA-acquired images. ${ }^{5-8}$ A detailed analysis is obtained with the help of virtual histology, using a color code for the different components of the plaque: white for calcium, red for the necrotic core, light green for the fibrolipidic core, dark green for fibrous tissue. The final results are available in absolute values or as a proportion in the plaque. ${ }^{9,10}$

The implantation of bioresorbable stents requires a careful lesion assessment to determine the need for and extent of lesion preparation, and to select the size and length of the device. The use of conventional imaging as well as preand post-procedural intracoronary imaging techniques is encouraged to optimize the implantation and the subsequent monitoring of the device. ${ }^{11}$

Given their structure and mechanical properties, compared to the pharmacologically-treated metallic stents, bioresorbable scaffolds require a meticulous evaluation of the lesion and also the frequent use of pre- and post-dilatation. As a result, the duration of the procedure and the use of contrast substance increases.

It is recommended to dilate the lesion with a noncompliant balloon before BVS implantation, by targeting balloons with a diameter corresponding to the estimated diameter of the reference vessel. Orthogonal angiographic projections are required for viewing the entire expansion of the balloon, otherwise it is necessary to use other preparation techniques before implanting the BVS. ${ }^{12}$ As a technical notion, pre-dilatation should be performed with a balloon of a size that is appropriate for the vascular diameter (ratio of 1:1). BVS should not be implanted in lesions that present suboptimal results after pre-treatment. If pre-dilatation results are not satisfactory, implantation of the bioresorbable stent will cause incomplete expansion associated with an increased risk for restenosis or in-stent thrombosis. ${ }^{13}$

Post-dilatation with a non-compliant high-pressure balloon should be performed routinely with a balloon diameter that exceeds the diameter of the device with a maximum of $0.5 \mathrm{~mm}$. The expansive limit of the bioresorbable scaffold is $0.5 \mathrm{~mm}$ above the nominal diameter. A $3.2 \mathrm{~mm}$ bioresorbable stent should not be dilated above $3.5 \mathrm{~mm}$, which is the size when the stent struts are prone to fracture. If the stent struts rupture, they will lead to subsequent complications that pose a potential cause of concern. ${ }^{12}$

Magnesium alloy bioresorbable stents present high radial force, which could expose it to fractures during expansion. ${ }^{14}$ Although the radial force of bioresorbable stents has been reported to be comparable to that of metallic stents, this holds true if the bioresorbable stent is expanded within the limits of its size. If the bioresorbable stent is expanded beyond its size, it can lose its radial force and possibly fracture. ${ }^{15}$

The topic of implantation methodology and subsequent follow-up of bioresorbable scaffolds is still largely discussed in the field of interventional cardiology. Therefore, this study aims the prospectively follow-up patients with significant coronary atherosclerotic plaques who have been treated electively with BVS implantation, via coronary CTA evaluation.

\section{MATERIALS AND METHODS}

We performed a prospective observational study, which included 30 patients with stable and unstable angina, who underwent elective BVS implantation for significant coronary atherosclerotic lesions in the CardioMed Medical Center between January 2015 and March 2017. Data regarding the patients' medical history and clinical examination were collected, and the patients presented at 1,6 , or 12 months for follow-up visits.

Other inclusion criteria were age over 18 years, BMI $<40 \mathrm{~kg} / \mathrm{m}^{2}$, signed written informed consent given by the patient. Exclusion criteria included STEMI or non-STEMI diagnosis, electric and hemodynamic instability at admission, known allergy for contrast agent, indication for long-term anticoagulant treatment, chronic kidney disease stage 4 or 5 , end-stage disease with life expectancy below 1 year. 


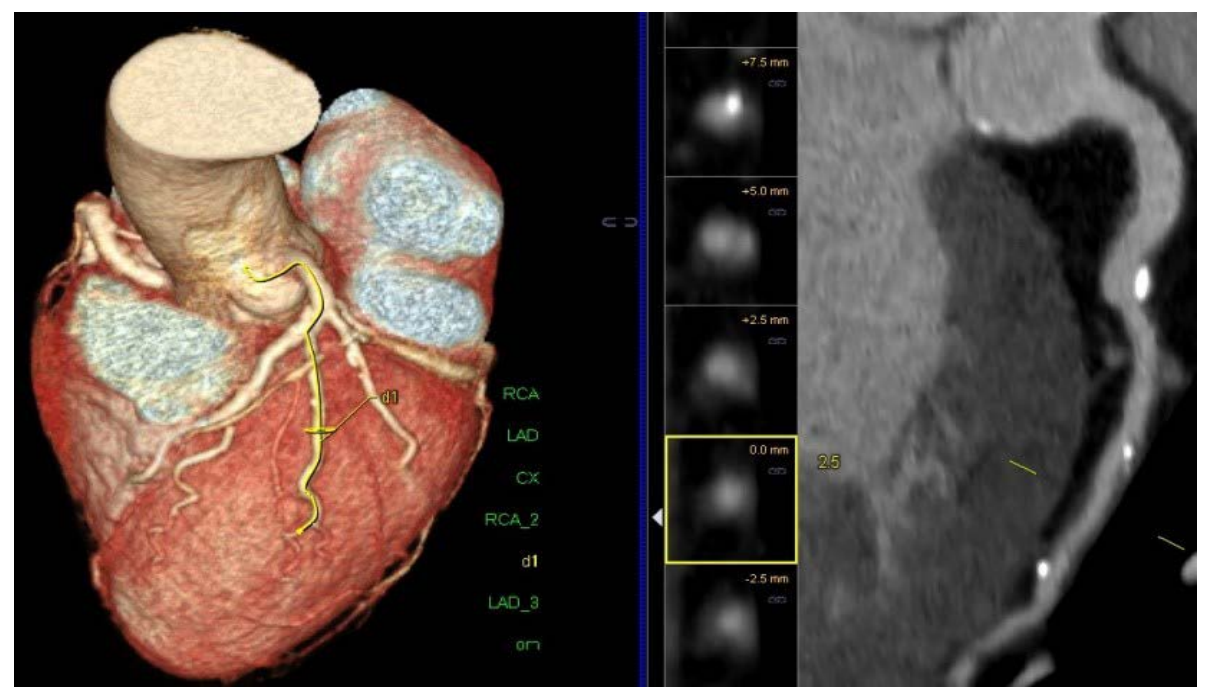

FIGURE 1. CTA of diagonal coronary artery 12 months after PTCA with BVS - no significant stenosis between BVS markers

All patients underwent CTA evaluation 12 to 24 months after PTCA in order to assess the morphological and virtual histology aspects of the coronary plaques. CTA was performed before and after BVS implantation for 16 patients and only after BVS implantation for 14 of the study subjects. In total, 48 coronary plaques were analyzed.
In order to evaluate the impact determined by the preand post-implantation procedures on the structure and morphology of the coronary artery, statistical analysis was performed in 2 subgroups, as follows:

- subgroup 1 - pre-implantation lesions $(n=48)$

- subgroup 2 - post-implantation lesions $(n=48)$

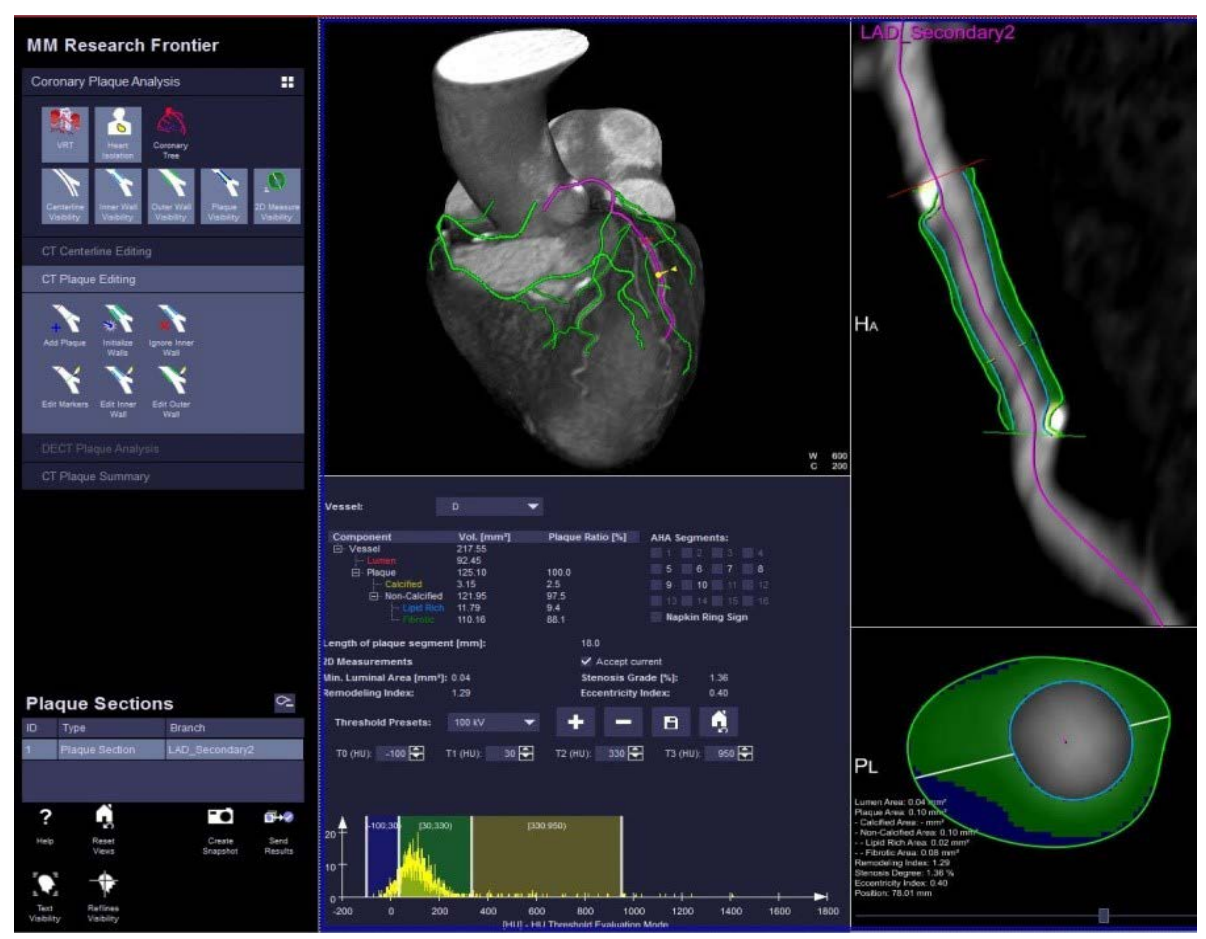

FIGURE 2. CTA of diagonal artery 12 months after PTCA with BVS, post-processed image with Syngo.via Frontier (Siemens AG, Erlangen, Germany) - no significant stenosis between BVS markers. 


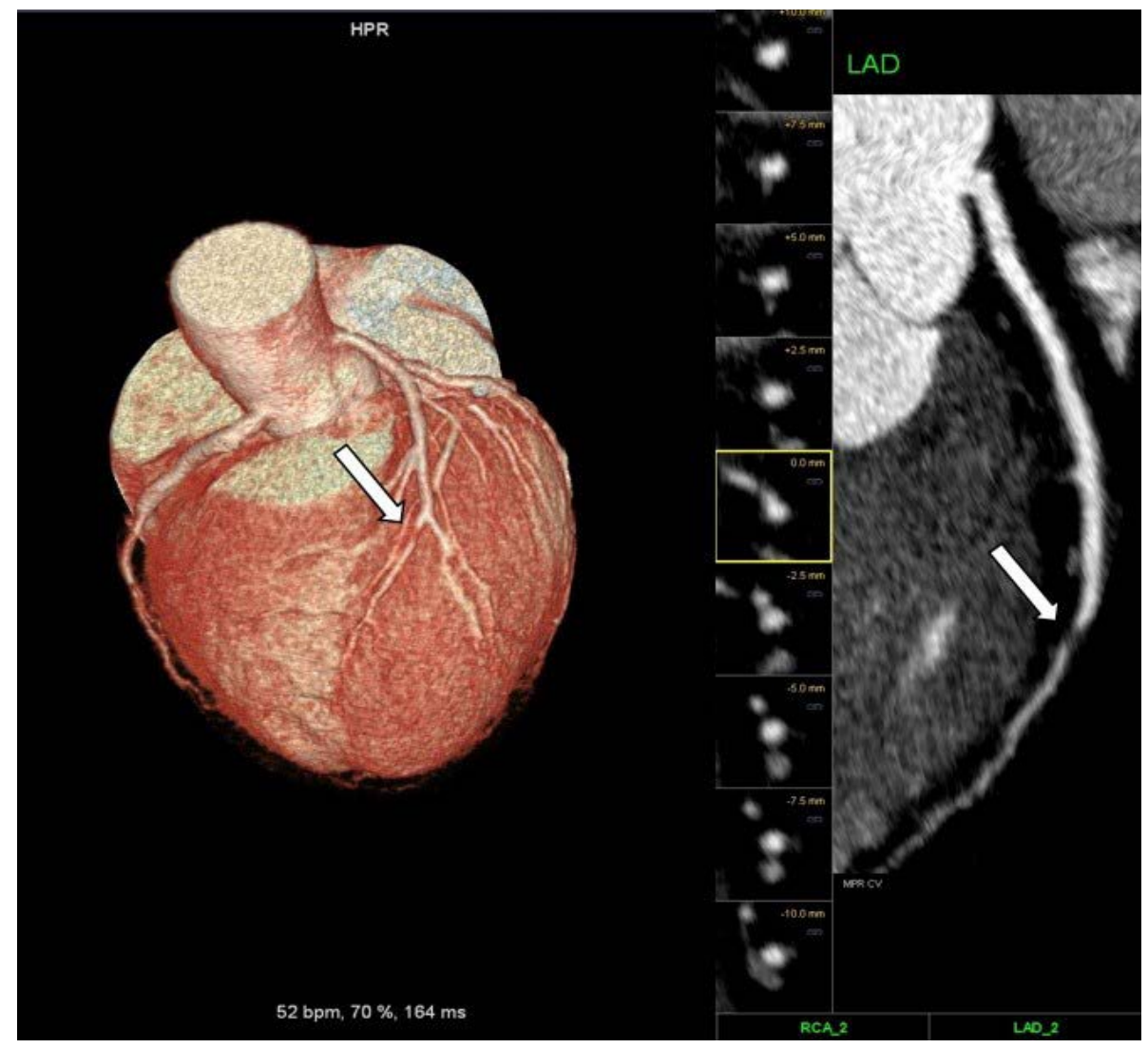

FIGURE 3. 128-slice CTA. Left anterior descending artery with critical stenosis in the third segment, before implantation of BVS

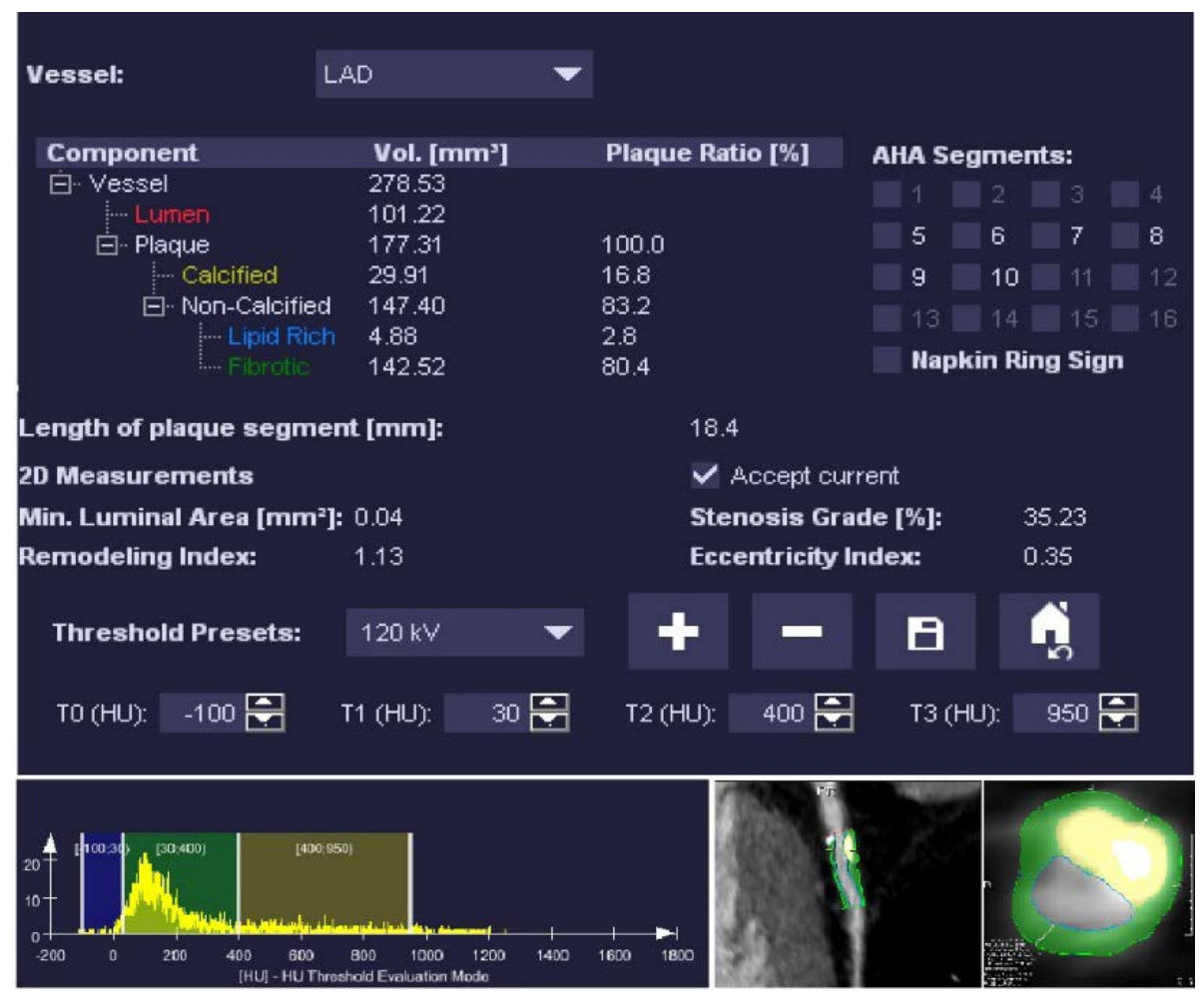

FIGURE 4. Coronary plaque analysis platform - Syngo.via Frontier software 


\section{CT scanning protocol}

CTA evaluations were performed in the Laboratory of Advanced Research in Cardiac Multimodal Imaging of the Cardio Med Medical Center of Tîrgu Mureş, with a 64- and 128-slice dual-source CT (SOMATOM Definition, Siemens Healthcare), with the following technical characteristics: $64 \times 0.5 \mathrm{~mm}$ and $128 \times 0.6 \mathrm{~mm}$ detector rows, $330 \mathrm{~ms}$ rotation time, and a table feed of $0.2-0.4 \mathrm{~mm}$ per rotation.

Optimization of the CT scanning protocol consisted in a fasting period of 5-24 h prior to CT examination, avoidance of smoking, caffeine use or physical exercises before CT scanning. During CT image acquisition, a heart rate $<60 \mathrm{bpm}$ was obtained with the oral titrated administration of Metoprolol or Ivabradine $1 \mathrm{~h}$ prior to the CT scan.

\section{Reconstruction of CT images and data analysis}

CT image post-processing was performed with a dedicated research software, Syngo.via Frontier, using a Siemens workstation (Siemens AG, Erlangen, Germany). Figure 1 presents a coronary angiographic 128-slice CT scan, Figure 2 presents a detailed analysis of a coronary plaque and the BVS, and Figure 3 presents a critical stenosis of the left anterior descending artery before implantation of the BVS.

\section{Plaque structure}

A coronary plaque analysis platform was used to assess morphology and plaque composition (Figure 4). Plaque structure was evaluated according to the CT density of its main components: lipids $(-100-30 \mathrm{HU})$, fibrotic tissue (30-400 HU) and calcifications (400-950 HU).

\section{Statistical analysis}

Statistical analysis was performed using GraphPad Prism 7 (GraphPad Software, San Diego, USA). Continuous variables were compared using Student's t-test for paired data or the Wilcoxon test when appropriate, and categorical data were compared using the Chi-square test. The statistical significance of the study was set at an alpha of 0.05 .

\section{Ethical approval}

This study was carried out in accordance with the World Medical Association's Declaration of Helsinki. All patients gave written informed consent, and the study protocol was approved by the Ethics Committee of the University of Medicine and Pharmacy of Tîrgu Mureș (approval no. 338/17.11.2017) and the Ethics Committee of CardioMed Medical Center (approval no. 29/28.12.2017).

\section{RESULTS}

\section{General characteristics of the study population}

The mean age of study population was $58.35 \pm 7.79$ years, and $20 \%$ of the study subjects were over 65 years. With regard to gender distribution, $86.66 \%$ of the subjects were males $(n=26)$.

General characteristics of the study population and their cardiovascular risk factors are presented in Table 1. In terms of left ventricular dysfunction incidence, $70 \%$ of the subjects presented a left ventricular ejection fraction $<55 \%, 63.33 \%$ were enrolled with a diagnosis of stable angina, and $36.67 \%$ were diagnosed with unstable angina.

After coronary CT evaluation, $43.33 \%$ of the subjects presented coronary artery disease (CAD) with lesions in a single vessel, $30 \%$ were diagnosed with 2 -vessel disease, and $26.66 \%$ presented severe 3-vessel disease. From the treated lesions, pre-dilatation was performed in $80 \%$ of cases, while post-dilatation was carried out in $26.66 \%$ of cases.

\section{Post-implantation CT analysis of coronary plaques}

Morphological and virtual histology aspects of the analyzed coronary plaques after BVS implantation are presented in Table 2.

After BVS implantation, a significant reduction was observed in the degree of stenosis $(61.63 \pm 12.63 \%$ in subgroup 1 vs. $24.41 \pm 12.48 \%$ in subgroup $2, \mathrm{p}<0.0001$ ) (Figure $5 \mathrm{~A})$ and the eccentricity index ( $0.46 \pm 0.24$ in subgroup 1 vs. $0.43 \pm 0.24$ in subgroup $2, p<0.0001$ ) (Figure $5 B$ ).

TABLE 1. General characteristics and cardiovascular risk factors

\begin{tabular}{lcc}
\hline & Mean value \pm SD & $\%$ \\
\hline Age, years & $58.35 \pm 7.79$ & \\
Age $>65$ years & 6 & 20 \\
Male gender & 26 & 86.66 \\
Female gender & 4 & 13.43 \\
EF $<55 \%$ & 21 & 70 \\
Hypertension & 25 & 80.64 \\
Creatinine clearance & $73.55 \pm 18.12$ & \\
Diabetes Mellitus & 6 & 20 \\
Dyslipidemia & 10 & 33.33 \\
History of AMl & 10 & 33.33 \\
\hline
\end{tabular}


TABLE 2. Morphological and virtual histology aspects of analyzed coronary plaques

\begin{tabular}{|c|c|c|c|}
\hline & $\begin{array}{c}\text { Pre-BVS } \\
\text { Mean value } \pm \text { SD }(95 \% \mathrm{Cl})\end{array}$ & $\begin{array}{c}\text { Post-BVS } \\
\text { Mean value } \pm \text { SD }(95 \% \mathrm{Cl})\end{array}$ & $p$ value \\
\hline Plaque length (mm) & $18.69 \pm 2.73(17.54-19.85)$ & $18.91 \pm 2.87(17.7-20.12)$ & 0.64 \\
\hline$\%$ stenosis & $61.63 \pm 12.63(52.02-66.69)$ & $23.41 \pm 12.48(18.14-28.68)$ & $<0.0001$ \\
\hline Eccentricity index & $0.46 \pm 0.24(0.36-0.57)$ & $0.43 \pm 0.24(0.33-0.53)$ & $<0.0001$ \\
\hline Remodeling index & $1.04 \pm 0.2(0.95-1.13)$ & $1.09 \pm 0.3(0.96-1.21)$ & 0.71 \\
\hline Vascular volume (mm3) & $277.7 \pm 86.36(241.3-314.2)$ & $283.7 \pm 72.24(253.2-314.2)$ & 0.79 \\
\hline Lumen volume (mm3) & $100.8 \pm 33.68(86.53-115.0)$ & $128.2 \pm 37.38(112.4-144.0)$ & 0.01 \\
\hline Dense calcium (mm3) & $13.59 \pm 17.36(6.26-20.92)$ & $16.07 \pm 19.0(8.0-24.09)$ & 0.45 \\
\hline Dense calcium (\%) & $6.72 \pm 7.79(3.43-10.02)$ & $8.98 \pm 9.0(5.15-12.82)$ & 0.25 \\
\hline Necrotic core $(\mathrm{mm} 3)$ & $169.8 \pm 63.7$ (142.9-196.7) & $143.6 \pm 53.62(121.0-166.2)$ & 0.12 \\
\hline Necrotic core (\%) & $93.27 \pm 7.79$ (89.98-96.56) & $91.0 \pm 9.0(87.17-94.84)$ & 0.25 \\
\hline Fibro-fatty (mm3) & $20.07 \pm 15.67(13.46-26.69)$ & $11.05 \pm 10.83(6.47-15.62)$ & 0.01 \\
\hline Fibro-fatty (\%) & $10.31 \pm 6.24(7.67-12.95)$ & $6.46 \pm 6.14(4.05-9.24)$ & 0.01 \\
\hline Fibrous (mm3) & $114.8 \pm 59.62(119.6-170.0)$ & $132.6 \pm 50.97$ (111.0-154.1) & 0.44 \\
\hline Fibrous (\%) & $82.97 \pm 7.74(79.69-86.24)$ & $84.26 \pm 10.73(79.73-88.79)$ & 0.63 \\
\hline Calcium score - local & $82.97 \pm 107.5(37.57-128.4)$ & $96.54 \pm 85.73(60.34-132.7)$ & 0.25 \\
\hline Calcium score - target coronary artery & $148.2 \pm 222.3(54.37-242.1)$ & $207.6 \pm 224.0(113.0-302.2)$ & 0.10 \\
\hline
\end{tabular}

In terms of plaque components, there were no significant differences between subgroup 1 and subgroup 2 regarding to the calcified volume $\left(13.59 \pm 17.36 \mathrm{~mm}^{3}\right.$ in subgroup 1 vs. $16.07 \pm 19.0 \mathrm{~mm}^{3}$ in subgroup $\left.2, \mathrm{p}=0.45\right), \%$ calcified $(6.72 \pm 7.79$ in subgroup 1 vs. $8.98 \pm 9.0$ in subgroup $2, p=$ $0.25)$, necrotic core volume ( $169.8 \pm 63.7 \mathrm{~mm}^{3}$ in subgroup 1 vs. $143.6 \pm 53.62 \mathrm{~mm}^{3}$ in subgroup $2, \mathrm{p}=0.12$ ), necrotic core \% (93.27 \pm 7.79 in subgroup 1 vs. $91.0 \pm 9.0$ in subgroup $2, \mathrm{p}=0.25)$, fibrotic volume $\left(114.8 \pm 59.62 \mathrm{~mm}^{3}\right.$ in subgroup 1 vs. $132.6 \pm 50.97 \mathrm{~mm}^{3}$ in subgroup $2, \mathrm{p}=0.44$ ), or fibrotic \% (82.97 \pm 7.74 in subgroup 1 vs. $84.26 \pm 10.73$ in subgroup $2, \mathrm{p}=0.63)$, except for lipid volume and lipid $\%\left(20.07 \pm 15.67 \mathrm{~mm}^{3}\right.$ in subgroup 1 vs. $11.05 \pm 10.83 \mathrm{~mm}^{3}$ in subgroup $2, \mathrm{p}=0.01$ ) which presented a significant reduction after BVS implantation (Figure 6).

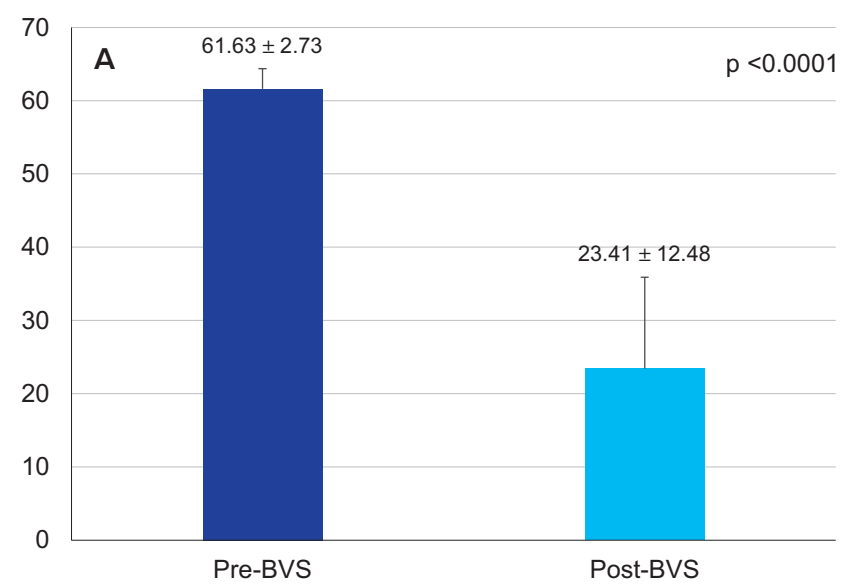

Regarding the calcium score evaluated locally (82.97 \pm 107.5 in subgroup 1 vs. $96.54 \pm 85.73$ in subgroup $2, \mathrm{p}=$ $0.25)$, at the level of the target coronary artery (148.2 \pm 222.3 in subgroup 1 vs. $206.6 \pm 224.0$ in subgroup $2, p=$ $0.10)$, and the total calcium score $(377.6 \pm 459.5$ in subgroup 1 vs. $529.5 \pm 512.9$ in subgroup $2, \mathrm{p}=0.32$ ), there were no significant differences when comparing lesions with versus without post-dilatation (Figure 7).

Table 3 presents the comparative analysis between the pre- and post-BVS implantation aspects of the CTA evaluation combined with the image post-processing analysis in terms of CT vulnerability markers of the analyzed coronary plaques.

Regarding CT vulnerability markers, the study groups presented significant differences only in terms of spotty

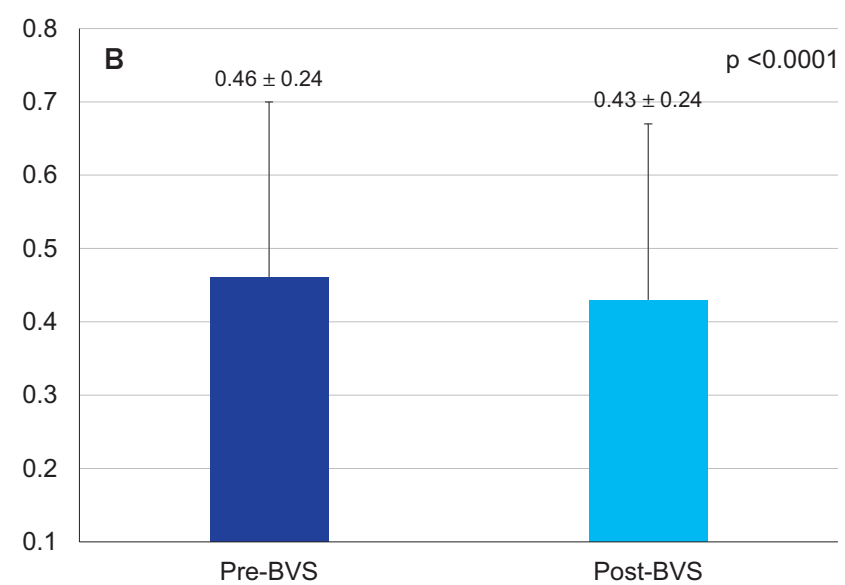

FIGURE 5. A - \% stenosis mean value pre- and post-BVS implantation; B - Eccentricity index pre- and post-BVS implantation 

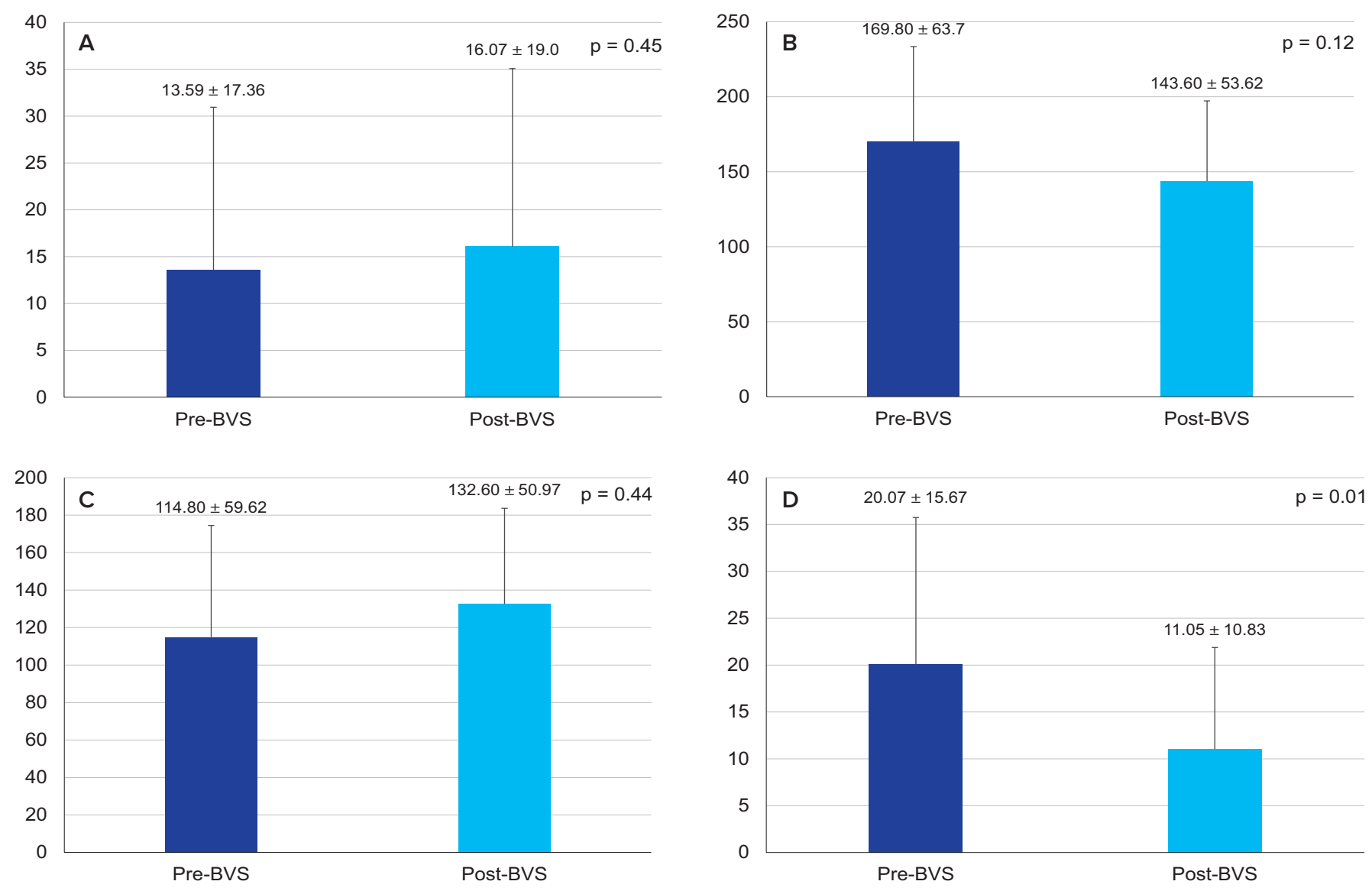

FIGURE 6. A - Calcified volume $\left(\mathrm{mm}^{3}\right)$; $\mathbf{B}-$ Necrotic core volume $\left(\mathrm{mm}^{3}\right) ; \mathbf{C}-$ Fibrotic volume $\left(\mathrm{mm}^{3}\right) ; \mathbf{D}-$ Lipid volume $\left(\mathrm{mm}^{3}\right)$

calcifications ( $66.66 \%$ in subgroup 1 vs. $79.16 \%$ in subgroup $2, \mathrm{p}=0.05)$ and low attenuation (37.5\% in subgroup 1 vs. $20.83 \%$ in subgroup $2, \mathrm{p}=0.01$ ) (Figure 8 ).

\section{DISCUSSIONS}

Given that coronary atherosclerotic plaques, treated by implantation of bioresorbable scaffolds, can also be evaluated after the procedure using a noninvasive method, this could lead to the routine evaluation of these devices through coronary CT angiography. This way, the coronary CT angiography evaluation of BVS can highlight certain complications that may occur over time..$^{16,17}$

The CTA evaluation of BVS is possible due to the special advantages of these structures, namely polymer resorption over time. All patients included in this study underwent BVS implantation, with a composition based on polymers. Regarding the radiological advantages, the radiotransparency of the device is worth mentioning, which facilitates the acquisition of coronary CT angiography, as well as subsequent image post-processing. ${ }^{17-20}$

The post-processing software dedicated to the detailed analysis of atherosclerotic plaque components enabled the analysis of important elements for a percutaneous procedure.

Another crucial element in a successful revascularization treatment using bioresorbable stents is the careful analysis of the target lesion. This thorough analysis, corroborated with noninvasive pre-interventional CT imaging, can contribute to the best revascularization approach of the atherosclerotic lesions.

Pre-dilatation techniques, implantation of the device, and its subsequent optimization by post-dilatation were elements evaluated in this study. When the first BVS had been implanted, there were no precise guidelines related to these procedural aspects; nevertheless, we monitored these aspects in this study. Although statistically significant differences were not obtained, differences in absolute values were observed for multiple parameters such as stenosis \% (CT performed 12 to 24 months postimplantation showed less procedural stenosis in patients with pre-dilatation); the eccentricity index, the remodeling index, vascular volume, plaque volume, and necrotic core were lower among these patients. Following the in- 

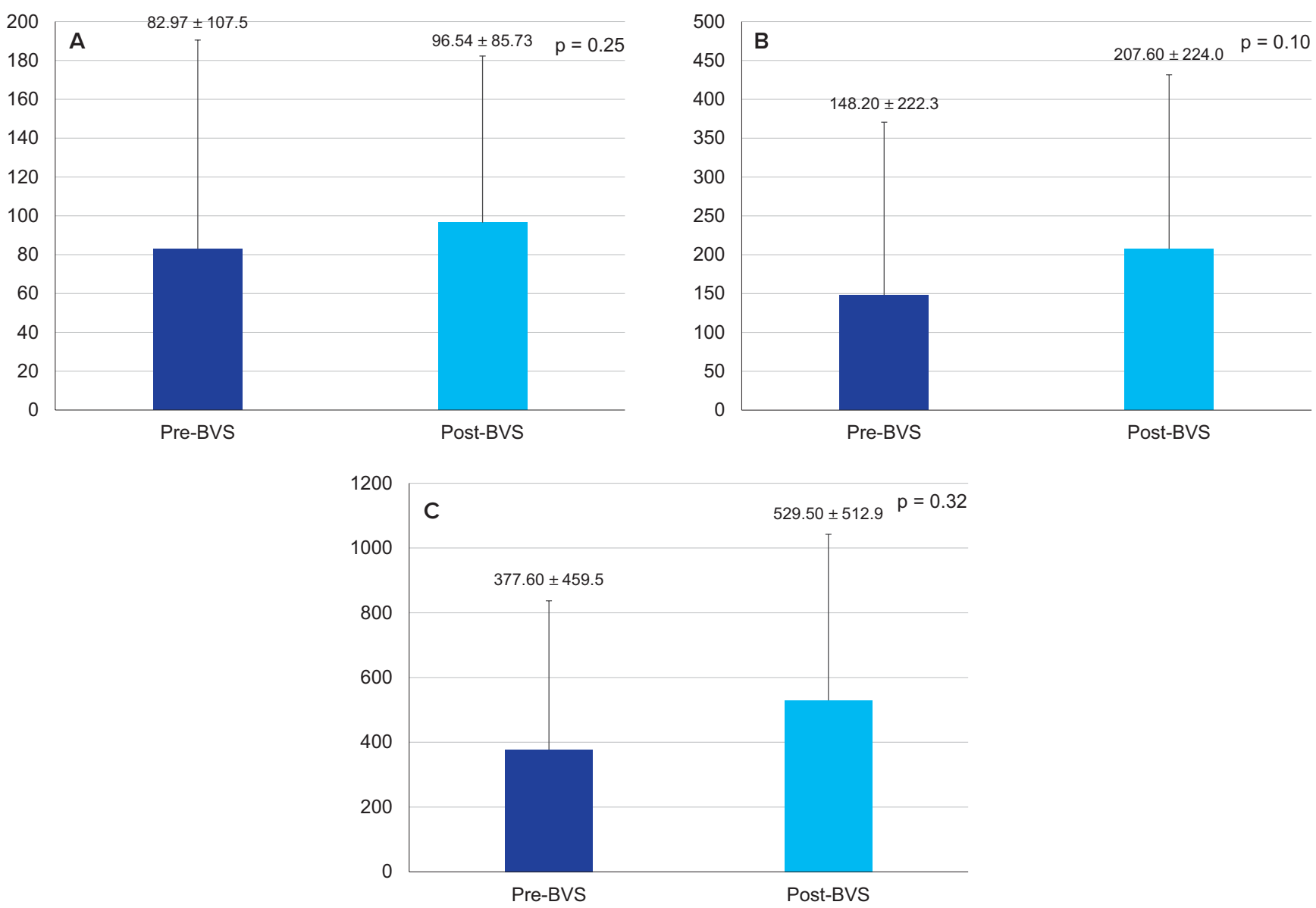

FIGURE 7. A - Local calcium score; B - Target lesion calcium score; C - Total calcium score

tervention, stent lumen was larger in patients with predilatation.

Regarding patients who underwent post-dilatation of the bioresorbable stent versus patients without post-dilatation, except for statistically significant differences in vascular volume and lumen volume, differences in absolute stent-stenosis values were observed, which were lower in stents that have been post-dilated.

No randomized trials with specific, mandatory, predefined implantation techniques have been completed, based on the PSP concept (pre-dilation, dimensioning, and post-dilatation of the bioresorbable stent). ${ }^{21}$ In this re-

TABLE 3. CT vulnerability markers

\begin{tabular}{lccc}
\hline & Pre-BVS (\%) & Post-BVS (\%) & p value \\
\hline Positive remodeling & 45.83 & 58.33 & 0.08 \\
Spotty calcifications & 66.66 & 79.16 & 0.05 \\
Napkin-Ring sign & 0 & 4.16 & - \\
Low attenuation & 37.5 & 20.83 & 0.01 \\
\hline
\end{tabular}

spect, the evaluation of these procedures can lead to new ideas, improvement, and mandatory implementation of certain specific techniques in the treatment of coronary atherosclerotic lesions with bioresorbable stents. It is recommended that the stents be implanted by trained and experienced operators in interventional cardiology. ${ }^{22-24}$

\section{CONCLUSIONS}

During the analysis of coronary artery plaques after the implantation of bioresorbable scaffolds with noninvasive CT imaging techniques, significant changes were noted both in the morphology of the atherosclerotic plaques treated with these devices and in the lumen and coronary wall. The assessment of coronary atherosclerotic plaques and bioresorbable scaffolds via coronary CT angiography could become a novel follow-up method for these cases.

\section{CONFLICT OF INTEREST}

Nothing to declare. 

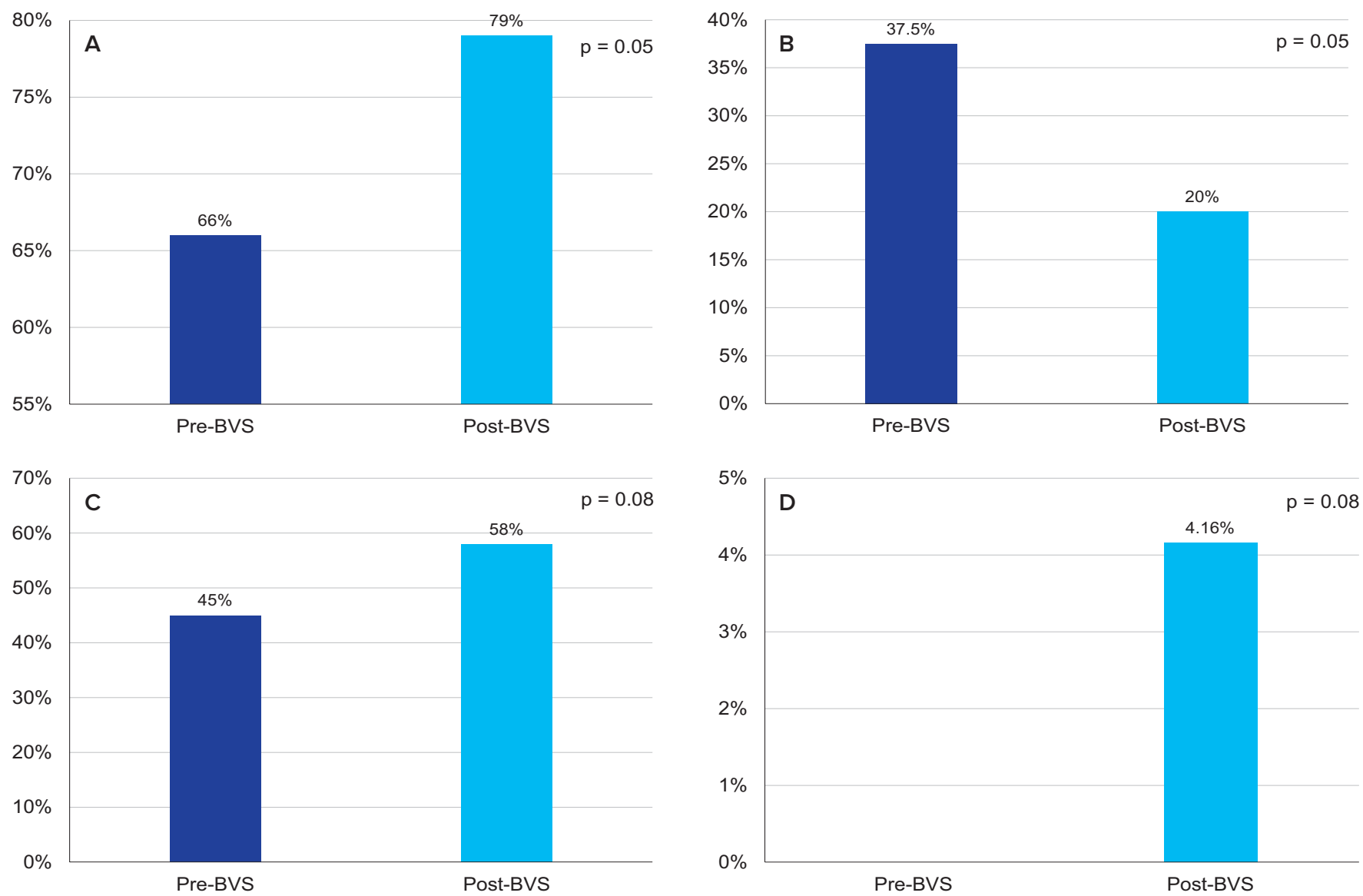

FIGURE 8. A - Spotty calcifications; B - Low attenuation; C - Positive remodeling; D - Napkin-ring sign

\section{ACKNOWLEDGEMENT}

This research was supported via the research grant no. 103544/2016, contract number 26/01.09.2016, entitled "Increasing the research capacity in the field of vulnerable plaque imaging, based on advanced nanoparticles, fusion imaging and computational simulation - PlaqueImage", financed by the Romanian Ministry of European Funds, the Romanian Government and the European Union.

\section{REFERENCES}

1. Onuma $Y$, Dudek D, Thuesen L, et al. Five-year clinical and functional multislice computed tomography angiographic results after coronary implantation of the fully resorbable polymeric everolimus-eluting scaffold in patients with de novo coronary artery disease: the ABSORB cohort A trial. JACC Cardiovasc Interv. 2013;6:999-1009.

2. Maeng M, Tilsted HH, Jensen LO, et al. Differential clinical outcomes after year versus 5 years in a randomised comparison of zotarolimus-eluting and sirolimus-eluting coronary stents (the SORTOUT III study): a multicentre, open-label, randomised superiority trial. Lancet. 2014;383:2047-2056.

3. Stone GW, Gao R, Kimura T, et al. 1-Year outcomes with the Absorb bioresorbable scaffold in patients with coronary artery disease: a patientlevel, pooled meta-analysis. Lancet. 2016;387:1277-1289.

4. Little WC, Constantinescu M, Applegate RJ, et al. Can coronary angiography predict the site of a subsequent myocardial infarction in patients with mild-to-moderate coronary artery disease? Circulation. 1988;78:1157-1166

5. Benedek T, Bucur O, Pașcanu I, Benedek I. Analysis of coronary plaque morphology by 64 multislice computed tomography coronary angiography and calcium scoring in patients with type 2 diabetes mellitus. Acta Endocrinologica. 2011;7:59-68.

6. Benedek I, Chitu M, Kovacs I, Bajka B, Benedek T. Incremental Value of preprocedural Coronary Computed Tomographic Angiography to classical Coronary Angiography for prediction of $\mathrm{PCl}$ complexity in left main stenosis. World Journal of Cardiovascular Disease. 2013;9:573-580.

7. Benedek T, Jako B, Benedek I. Plaque quantification by coronary CT and intravascular ultrasound identifies a low CT density core as a marker of plaque instability in acute coronary syndromes. Int Heart J. 2014:55:22-28.

8. Benedek T, Gyongyosi M, Benedek I. Multislice computed tomographic coronary angiography for quantitative assessment of culprit lesions in acute coronary syndromes. Can J Cardiol. 2013;29:364-371.

9. Gogas BD, Serruys PW, Diletti R, et al. Vascular response of the segments adjacent to the proximal and distal edges of the ABSORB everolimuseluting bioresorbable vascular scaffold: 6-month and 1-year follow-up assessment: a virtual histology intravascular ultrasound study from the first-in-man ABSORB cohort B trial. JACC Cardiovasc Interv. 2012;5:656665.

10. Verheye S, Martinet W, Kockx MM, et al. Selective clearance of macrophages in atherosclerotic plaques by autophagy. J Am Coll Cardiol 2007:49:706-715

11. Ferent IF, Mester A, Hlinomaz $O$, et al. Intracoronary Imaging for Assessment of Vascular Healing and Stent Follow-up in Bioresorbable Vascular Scaffolds. Current Medical Imaging Reviews. 2018. [E-pub ahead of print.] doi: 10.2174/1573405614666180604093621.

12. Fajadet $\mathrm{J}$, Haude $\mathrm{M}$, Joner $\mathrm{M}$, et al. Magmaris preliminary recommendation upon commercial launch: a consensus from the expert panel on 14 April 2016. Eurolntervention. 2016;12:828-833. 
13. Capodanno D, Gori T, Nef H, et al. Percutaneous coronary intervention with everolimus-eluting bioresorbable vascular scaffolds in routine clinical practice: early and midterm outcomes from the European multicentre GHOST-EU registry. Eurolntervention. 2015;10:1144-1153.

14. Onuma $Y$, Serruys PW. Bioresorbable scaffold: the advent of a new era in percutaneous coronary and peripheral revascularization? Circulation. 2011;123:779-797.

15. Ormiston JA, De Vroey F, Serruys PW, Webster MW. Bioresorbable polymeric vascular scaffolds: a cautionary tale. Circ Cardiovasc Interv. 2011:4:535-538

16. Bourantas CV, Garcia-Garcia HM, Diletti R, Muramatsu T, Serruys PW. Early detection and invasive passivation of future culprit lesions: a future potential or an unrealistic pursuit of chimeras? Am Heart J. 2013;165:869881.

17. Brugaletta S, Heo JH, Garcia-Garcia HM, et al. Endothelial-dependent vasomotion in a coronary segment treated by ABSORB everolimus-eluting bioresorbable vascular scaffold system is related to plaque composition at the time of bioresorption of the polymer: indirect finding of vascular reparative therapy? Eur Heart J. 2012;33:1325-1333.

18. Brugaletta S, Radu MD, Garcia-Garcia HM, et al. Circumferential evaluation of the neointima by optical coherence tomography after ABSORB bioresorbable vascular scaffold implantation: can the scaffold cap the plaque? Atherosclerosis. 2012;221:106-112.

19. Gomez-Lara J, Brugaletta S, Farooq V, et al. Angiographic geometric changes of the lumen arterial wall after bioresorbable vascular scaffolds and metallic platform stents at 1-year follow-up. JACC Cardiovasc Interv. 2011;4:789-799

20. Lane JP, Perkins LE, Sheehy AJ, et al. Lumen gain and restoration of pulsatility after implantation of a bioresorbable vascular scaffold in porcine coronary arteries. JACC Cardiovasc Interv. 2014;7:688-695.

21. Serruys PW, Onuma Y. Dmax for sizing, PSP-1, PSP-2, PSP-3 or OCT guidance: interventionalist's jargon or indispensable implantation techniques for short- and long-term outcomes of Absorb BRS? Eurolntervention. 2017;12:2047-2056.

22. James SK, Stenestrand U, Lindback J, et al. Long-term safety and efficacy of drug-eluting versus bare-metal stents in Sweden. $N$ Engl J Med 2009:360:1933-1945

23. Lagerqvist B, James SK, Stenestrand $U$, et al. Long-term outcomes with drug-eluting stents versus bare-metal stents in Sweden. N Engl J Med. 2007;356:1009-1019.

24. Serruys PW, Daemen J. The SCAAR registry or the Swedish yo-yo. Eurolntervention. 2007;3:297-300 